\title{
I| reclamo e la mediazione tra procedimento e processo
}

Sommario: 1. Collocazione e fondamento normativo - 2. Natura giuridica. 3 . SAmbito oggettivo di applicazione - 4. Ambito soggettivo di applicazione. -5 . Organo competente a conoscere della procedura -6 . Modalità procedurali -7 . Effetti del mancato accoglimento dell'istanza - 8. Perfezionamento dell'accordo di mediazione.

1. Il reclamo e la mediazione tributaria ${ }^{1}$ si collocano nella fase immediatamente precedente all'instaurazione del processo tributario, giudizio che consente al

\footnotetext{
* Salvatore Antonello Parente - dottore di ricerca in "Diritto tributario" (XXVI ciclo) - Dipartimento di Giurisprudenza - Università degli Studi di Bari "Aldo Moro" - e-mail: salvo.par@live.it.

${ }^{1}$ In materia, cfr. M. Scuffi, Gli istituti deflattivi del contenzioso tributario secondo la manovra "correttiva" del 2011, in Il fisco, n. 47/2011, p. 7641 ss.; V. Busa, Le nuove prospettive della mediazione tributaria, in Corr. trib., n. 11/2012, p. 765 ss.; M. Busico, L'ambito di operatività del reclamo e della mediazione: i limiti oggettivi, soggettivi e quantitativi, in Corr. trib., n. 10/2012, p. 704 ss.; S. Capolupo, Mediazione tributaria e accertamento con adesione, in Corr. trib., n. 8/2012, p. 584 ss.; A. Carinci, Perduranti profili di criticità della presentazione del reclamo, in Corr. trib., n. 37/2012, p. 2877 ss.; M. Chionchio, Z. Gola e R. Lupi, Mediazione tributaria: irragionevolezze vere e presunte sulla via della "deprocessualizzazione", in Lo Stato civile italiano, n. 2/2013, p. 194 ss.; G. Corasaniti, Commento all'art. 17-bis, in C. Consolo e C. Glendi, Commentario breve alle leggi del processo tributario, Padova, 2012, p. 236 ss.; M. Di Pirro, La mediazione tributaria, Napoli, 2012; V. Ficari, Brevi note sulla mediazione fiscale, in Boll. trib., n. 19/2012, p. 1364 ss.; A. Giovannini, Giurisdizione condizionata e reclamo amministrativo, in Riv. trim. dir. trib., 2012, p. 915 ss.; I. Lucati, Il filtro della mediazione obbligatorio anche per le liti tributarie, in La Responsabilità Civile, n. 5/2012, p. 396 ss.; A. Guidara e D. Stevanato, Mediazione fiscale: un provvedimento improvvisato su una strada giusta, in Dial. trib., 2012, 1, p. 92 ss.; C. Maiorano, La mediazione fiscale tra intuizione e realtà, in Boll. trib., 2012, p. 85 ss.; M. Martis, Reclamo e mediazione fiscale. Un nuovo strumento di convergenza tra gli interessi del cittadino e della pubblica amministrazione, in Riv. dir. trib., n. 9/2012, pt. I, p. 809 ss.; F. Pistolesi, Il reclamo e la mediazione nel processo tributario, in Rass. trib., n. 1/2012, p. 65 ss.; F. Pistolesi, Ambito applicativo della mediazione tributaria e sospensione della riscossione, in Corr. trib., n. 19/2012, p. 1429 ss.; G. Sepio, Mediazione tributaria e sanzioni nel coordinamento
} 
contribuente di impugnare un provvedimento illegittimo dell'amministrazione finanziaria, ai fini del suo annullamento.

Si tratta di istituti deflattivi del contenzioso, disciplinati dall'art. 17 bis, d.lgs. n. 546/1992, sintomatici dell'evoluzione del sistema normativo verso una "marginalizzazione" (rectius disincentivo) ${ }^{2}$ della fase contenziosa, da intendersi quale extrema ratio, derubricata a mera e remota eventualità, risolvibile attraverso procedure di soluzione dei conflitti strutturate in modo tale da risultare convenienti e appetibili per entrambe le parti ${ }^{3}$.

Fin dall'introduzione di tali strumenti, sono emerse una serie di criticità, culminate in una nota pronuncia della Consulta ${ }^{4}$, a causa del ritardo provocato nell'accesso alla tutela giurisdizionale ${ }^{5}$.

Il reclamo/mediazione assurge a procedura obbligatoria, in presenza di taluni requisiti oggettivi e soggettivi, finalizzata ad esaminare, quasi come filtro preventivo, la fondatezza dei motivi del ricorso e la legittimità della pretesa fiscale. La figura, inoltre, mira a verificare se sia possibile evitare, mediante l'accoglimento del reclamo o della proposta di mediazione, che la controversia prosegua in sede giurisdizionale ${ }^{6}$.

Questi istituti sono espressione del rapporto di collaborazione ed affidamento esistente tra i due soggetti del rapporto giuridico d'imposta: l'amministrazione finanziaria si sveste dei panni di controllore per indossare quelli di interlo-

con gli altri istituti deflativi del contenzioso, in Corr. trib., n. 36/2012, p. 2790 ss.; A. Turchi, voce Reclamo e mediazione nel processo tributario, in Dig. disc. priv., sez. comm., Aggiornamento, vol. 6, Torino, 2012, p. 615 ss.; C. Attardi, Reclamo e mediazione: costituzionalità e ricadute sulla teoria generale del processo tributario, in Corr. trib., n. 18/2013, p. 1446 ss.; G. Corasaniti, Il reclamo e la mediazione nel sistema tributario, Padova, 2013; E. De Mita, Il reclamo tributario limita i diritti del contribuente, in Il Sole 24 Ore, 22 settembre 2013, p. 25; V. Ficari, Possibili scenari futuri per la mediazione tributaria, in Corr. trib., n. 40/2013, p. 3187 ss.; A. Giovannini, Reclamo e mediazione tributaria: per una riflessione sistematica, in Rass. trib., n. 1/2013, p. 51 ss.; M. Montanari, Il processo tributario nel segno della mediazione, in Dir. e prat. trib., n. 1/2013, pt. I, p. 153 ss.; G. Pizzonia, Reclamo e mediazione tributaria: deflazione del contenzioso o inflazione di procedimenti?, in Riv. dir. fin., n. 1/2013, p. 71 ss.; R. Serrentino, Dubbi di costituzionalità del reclamo e della mediazione tributaria, in Riv. trim. dir. trib., n. 4/2013, p. 929 ss.; A. Magliaro, Reclamo e mediazione tributaria: il ruolo del Garante del contribuente, in La Rivista di Finanza, n. 2/2014, p. 39 ss.; R. Serrentino, Il "nuovo" istituto della mediazione tributaria: osservazioni critiche, in Dir. e prat. trib., n. 6/2013, pt. I, p. 1347 ss.; C. Gioè, Il reclamo e la mediazione nel giudizio tributario, Torino, 2015.

2 Cfr. M. Leo, La riforma del contenzioso tributario: cose fatte e cose da fare, in Il fisco, n. 42/2015, p. 4019.

${ }^{3}$ Cfr. M. Leo, Verso un ordinamento fiscale più moderno, con meno liti e più dialogo, in Il fisco, n. 29/2015, p. 2809.

${ }^{4}$ Corte Cost., 16 aprile 2014, n. 98, in G.U., 23 aprile 2014, n. 18 - $1^{\text {a }}$ Serie Speciale - Corte Costituzionale.

${ }^{5}$ Cfr. B. Bellè, Mediazione e reclamo: due istituti inutili, in Riv. dir. trib., n. 10/2012, pt. I, p. 863 ss.

${ }^{6}$ Cfr. Circolare Agenzia delle Entrate, 29 dicembre 2015, n. 38/E, in www.agenziaentrate.gov. it, p. 30 . 
cutore, aperto ed attento al dialogo, che, «consapevole della complessità della realtà economica, entro cui muovono gli operatori, e delle incertezze generate da un sistema normativo pletorico ed incoerente, tenta di proporsi alla stregua di un vero e proprio "consulente istituzionale" delle imprese» ${ }^{7}$; in questa luce, contribuente e fisco possono "accordarsi" in ordine alle modalità di applicazione delle norme tributarie, notoriamente complesse e mutevoli, calandole in contesto economico in continuo divenire ${ }^{8}$.

2. Com'è noto, per le controversie, ricomprese nella giurisdizione tributaria, il cui valore non ecceda i 20.000,00 euro, è richiesta, ai fini della procedibilità della domanda, la preventiva proposizione di un reclamo, che può anche contenere una proposta di mediazione, volta a dirimere l'insorgenda lite; in caso di mancato accoglimento del reclamo o della proposta di mediazione, si producono gli effetti propri del ricorso tributario?.

Dunque, la proposizione del reclamo dà vita ad una fase preliminare di natura amministrativa volta ad evitare la potenziale lite, mediante un dialogo tra i due soggetti del rapporto giuridico d'imposta: l'esame congiunto della fattispecie, unitamente all'analisi delle questioni giuridiche controverse, è finalizzato a risolvere la lite amichevolmente, evitando le spese e l'alea di un procedimento giurisdizionale ${ }^{10}$.

In altri termini, «con la procedura amministrativa del reclamo l'Amministrazione finanziaria è chiamata a svolgere non già una funzione di amministrazione attiva, bensì un'attività di riesame giustiziale dell'atto impositivo oggetto di reclamo ${ }^{11}$.

Il procedimento, inserito a pieno titolo tra gli strumenti alternativi di risoluzione delle controversie, consente di conciliare gli interessi del contribuente con quelli dell'amministrazione finanziaria: il primo, a seguito dell'esperimento dell'istituto, può vedere modificata, in maniera a lui favorevole, la pretesa fiscale; l'ufficio fiscale, invece, ha l'occasione di approfittare di questo momento per ritornare sui propri passi, correggere gli errori e valutare al meglio dati ed elementi che, in fase contenziosa, potrebbero compromettere l'intera pretesa impositiva ${ }^{12}$.

M. Leo, Verso un ordinamento fiscale più moderno, con meno liti e più dialogo, cit., p. 2807-2808.

${ }^{8}$ Cfr. M. Leo, Verso un ordinamento fiscale più moderno, con meno liti e più dialogo, cit., p. 2808.

9 Cfr. M. Conigliaro, Restyling di reclamo-mediazione e conciliazione giudiziale per puntare sugli strumenti deflattivi, in Il fisco, n. 31/2015, p. 3030.

${ }^{10}$ Cfr. S. La Rocca, Il reclamo e la mediazione nel "nuovo" processo tributario, in Boll. trib., n. 23/2015, p. 1694.

${ }^{11}$ G. Corasaniti, Il reclamo e la mediazione nel sistema tributario, cit., p. 46.

${ }_{12}$ Cfr. S. La Rocca, Il reclamo e la mediazione nel "nuovo" processo tributario, cit., p. 1700. 
3. Sotto il profilo oggettivo, l'obbligo del previo esperimento del reclamo ha ad oggetto le sole controversie, rientranti nella giurisdizione tributaria, definita dall'art. 2, d.lgs. n. 546/1992, il cui valore non sia superiore ad euro 20.000,00.

In relazione alle controversie di valore superiore a tale soglia, non è preclusa una definizione condivisa della lite, potenziale o reale, potendo le parti esperire forme di contraddittorio preventivo, oramai obbligatorie in diverse tipologie di accertamento, nonché, nel corso del giudizio, addivenire ad una conciliazione tributaria.

Il valore della lite è pari all'importo del tributo richiesto con il provvedimento impugnato, al netto di eventuali interessi e sanzioni, ovvero corrisponde all'entità delle sanzioni, qualora oggetto di contestazione sia un atto di irrogazione delle stesse $^{13}$; se la lite abbia ad oggetto il rifiuto (espresso o tacito) ad un'istanza di rimborso, la controversia sarà commisurata all'entità del tributo di cui viene chiesta la restituzione, al netto degli accessori, salvo che il rimborso concerna più periodi d'imposta, dovendosi, in tale ipotesi fare riferimento all'importo del tributo oggetto di rimborso per ogni periodo d'imposta ${ }^{14}$; in caso di impugnazione di taluni atti della riscossione, il valore della controversia è determinato sulla base dell'atto impugnato.

In coerenza con questa impostazione, il comma 1 dell'art. 17 bis, d.lgs. n. 546/1992, fissa una regola generale, escludendo dall'ambito di operatività del reclamo le controversie il cui valore sia indeterminabile ${ }^{15}$. Non mancano, tuttavia, eccezioni, atteso che l'ultimo inciso del medesimo comma impone l'obbligo del previo esperimento del reclamo in ordine al contenzioso catastale, che rientra nella giurisdizione tributaria per effetto dell'art. 2, comma 2, primo periodo, d.lgs. n. 546/1992. Trattandosi di un'eccezione alla regola generale, come tale di stretta interpretazione, non sarebbero suscettibili di reclamo le controversie, diverse da quelle catastali, aventi valore indeterminabile.

Del pari, per espressa previsione normativa, sono escluse dalla procedura de qua le controversie concernenti il recupero di aiuti di Stato di cui all'art. 47 bis, d.lgs. n. 546/1992 ${ }^{16}$.

${ }_{13}$ Cfr. M. Conigliaro, Nuove regole per reclamo e mediazione tributaria, ma la terzietà rimane una chimera, in Il fisco, n. 42/2015, p. 4021; V. Uckmar, Interventi sul contenzioso tributario, in Dir. e prat. trib., n. 6/2015, pt. I, p. 985.

${ }^{14}$ Cfr. S. La Rocca, Il reclamo e la mediazione nel "nuovo" processo tributario, cit., p. 1694-1695.

${ }_{15}$ Cfr. Circolare Agenzia delle Entrate, 29 dicembre 2015, n. 38/E, cit., p. 33; M. Conigliaro, Nuove regole per reclamo e mediazione tributaria, ma la terzietà rimane una chimera, cit., p. 4021; V. Uckmar, Interventi sul contenzioso tributario, cit., pt. I, p. 985.

${ }^{16}$ Cfr. Circolare Agenzia delle Entrate, 29 dicembre 2015, n. 38/E, cit., p. 33; S. La Rocca, Il reclamo e la mediazione nel "nuovo" processo tributario, cit., p. 1695; M. Conigliaro, Restyling di reclamo-mediazione e conciliazione giudiziale per puntare sugli strumenti deflattivi, cit., p. 3030; V. Uckmar, Interventi sul contenzioso tributario, cit., p. 985; M. Conigliaro, Nuove regole per reclamo e mediazione tributaria, ma la terzietà rimane una chimera, cit., p. 4021. 
4. Dal punto di vista soggettivo, l'ambito di applicazione del reclamo/mediazione concerne tutte le controversie rientranti nella giurisdizione tributaria, aventi ad oggetto l'impugnazione di atti e provvedimenti emessi da qualsiasi tipologia di ente impositore (Agenzia delle Entrate; Agenzia delle Dogane e dei Monopoli; Enti locali, per ciò che concerne i tributi locali propri o quelli dagli stessi amministrati; cancellerie o segreterie giudiziarie, in ordine al contributo unificato; agente della riscossione o concessionari privati di cui all'art. 53, d.lgs. n. 446/1997, in relazione agli atti emessi dagli stessi) ${ }^{17}$.

Peculiare appare l'applicazione del reclamo agli atti emessi dall'agente della riscossione o dai concessionari privati di cui all'art. 53, d.lgs. n. 446/1997'18: trattandosi di soggetti che non hanno la disponibilità del tributo, l'istituto troverà applicazione solo quando si voglia agire in giudizio per far valere i vizi propri della cartella di pagamento ovvero si voglia impugnare il provvedimento di fermo di beni mobili registrati o l'iscrizione di ipoteca sugli immobili ${ }^{19}$.

Dunque, per tutte le controversie tributarie di valore non superiore ad euro $20.000,00$, aventi ad oggetto atti emessi da qualsivoglia "ente impositore", è necessario esperire, in via preventiva e stragiudiziale, il reclamo/mediazione: in via esemplificativa, rientrano in questo regime tutti gli atti impugnabili dinanzi al giudice tributario, ai sensi dell'art. 19, d.lgs. n. 546/1992, quali l'avviso di accertamento, l'avviso di liquidazione, il ruolo, il provvedimento di irrogazione delle sanzioni, il diniego o la revoca di agevolazioni, il rifiuto (espresso o tacito) ad un'istanza di rimborso, gli atti della riscossione, come cartella di pagamento, avviso di intimazione, iscrizione di ipoteca su immobili, fermo di beni mobili registrati; inoltre, sono oggetto di reclamo gli atti emessi dall'Agenzia delle

${ }_{17}$ Cfr. S. La Rocca, Il reclamo e la mediazione nel "nuovo" processo tributario, cit., p. 1695-1696; M. Conigliaro, Restyling di reclamo-mediazione e conciliazione giudiziale per puntare sugli strumenti deflattivi, cit., p. 3030; V. Uckmar, Interventi sul contenzioso tributario, cit., p. 986; M. Conigliaro, Nuove regole per reclamo e mediazione tributaria, ma la terzietà rimane una chimera, cit., p. 4021; S. Mazzocchi, Mediazione tributaria, sospensione cautelare e controversie catastali: un pericoloso "intreccio" di norme, in Il fisco, n. 34/2015, p. 3220; U. Perrucci, Brevi annotazioni sulla nuova versione del processo tributario, in Boll. trib., n. 21/2013, p. 1475; M. Conigliaro, Luci ed ombre nella riforma del processo tributario, in Il fisco, n. 30/2015, p. 2931; C. Glendi, La riforma del contenzioso tributario, in Dir. e prat. trib., n. 5/2015, pt. I, p. 797; M. Villani, La parziale riforma del processo tributario, in Il tributo, n. 12/2015, p. 17; Circolare Agenzia delle Entrate, 29 dicembre 2015, n. 38/E, cit., p. 31.

${ }_{18}$ Cfr. M. Conigliaro, Luci ed ombre nella riforma del processo tributario, cit., p. 2931; V. Uckmar, Interventi sul contenzioso tributario, cit., p. 987. Per la disciplina vigente prima della novella, cfr. E. Fronticelli Baldelli, Effetti del reclamo/mediazione sulla posizione processuale dell'agente della riscossione, in Corr. trib., n. 13/2013, p. 1061 ss.; F. Brighenti, Cartella di pagamento: obbligo di mediazione? (Quando nelle circolari circolano idee confuse), in Boll. trib., n. 9/2014, p. 667.

${ }^{19}$ Cfr. Circolare Agenzia delle Entrate, 29 dicembre 2015, n. 38/E, cit., p. 32. Negli stessi termini, cfr. M. Conigliaro, Restyling di reclamo-mediazione e conciliazione giudiziale per puntare sugli strumenti deflattivi, cit., p. 3030; M. Conigliaro, Nuove regole per reclamo e mediazione tributaria, ma la terzietà rimane una chimera, cit., p. 4022; V. Uckmar, Interventi sul contenzioso tributario, cit., p. 987; M. Villani, La parziale riforma del processo tributario, cit., p. 17-18. 
dogane e dei monopoli, quali avvisi di pagamento per la riscossione delle accise, avviso di accertamento doganale, controversie concernenti le materie regolate dal Codice Doganale Comunitario, avviso suppletivo e di rettifica doganale, avviso di rettifica della dichiarazione doganale, bolletta doganale annotata, unitamente ad altri atti inerenti le operazioni doganali ${ }^{20}$; infine, sono assoggettati alla procedura de qua anche altre tipologie di atti tributari, come quelli emanati dagli Enti locali, in relazione ai tributi da essi amministrati, e adottati dagli uffici giudiziari in relazione al contributo unificato ovvero posti in essere da altri soggetti (ad es. consorzi di bonifica), accomunati dalla natura squisitamente tributaria ${ }^{21}$.

5. L'organo competente a conoscere della procedura appartiene alla stessa autorità che ha emanato l'atto impugnato ${ }^{22}$, ivi compresi gli agenti della riscossione ed i concessionari privati di cui all'art. 53, d.lgs. n. 446/1997, a cui il reclamo si applica solo ove compatibile; l'amministrazione emanante, però, vi provvede attraverso strutture diverse ed autonome da quelle che hanno curato l'istruttoria degli atti reclamabili ${ }^{23}$. Le Agenzie fiscali non hanno grosse difficoltà ad ottemperare alla disposizione normativa, a differenza degli enti locali, primi fra tutti i comuni, caratterizzati spesso da carenze strutturali ed organizzative ${ }^{24}$.

Secondo taluni ${ }^{25}$, tale circostanza potrebbe far venir meno la terzietà del soggetto chiamato a pronunciarsi sul reclamo, in quanto, l'organo preposto alla procedura, anche se autonomo, costituisce comunque un'articolazione dell'ente che ha emesso l'atto reclamato: il contribuente si ritroverebbe, dunque, a discutere

${ }^{20}$ Sulla questione, cfr. Circolare dell'Agenzia delle Dogane e dei Monopoli, 23 dicembre 2015, n. 21/D, in https://www.agenziadoganemonopoli.gov.it/portale/documents/20182/890996/ lgpa-c-20151223-21interpelli+versione+firmata.pdf/b4c38727-6129-4a25-8e7f-39aa19841d41.

${ }_{21}^{21}$ Cfr. S. La Rocca, Il reclamo e la mediazione nel "nuovo" processo tributario, cit., p. 1696.

${ }^{22}$ In passato, una soluzione alternativa, prospettata da F. D'ayala Valva, La Corte costituzionale preannuncia le ragioni di illegittimità costituzionale della mediazione tributaria, in Riv. dir. trib., n. 2/2013, pt. II, p. 100, proponeva di affidare al Garante del Contribuente, quale soggetto terzo ed imparziale, il compito di presiedere al procedimento di reclamo/mediazione.

${ }^{23}$ Cfr. Circolare Agenzia delle Entrate, 29 dicembre 2015, n. 38/E, cit., p. 37; M. Villani, La parziale riforma del processo tributario, cit., p. 17.

${ }^{24}$ Cfr. M. Conigliaro, Nuove regole per reclamo e mediazione tributaria, ma la terzietà rimane una chimera, cit., p. 4021-4022.

${ }_{25}$ Cfr. M. Conigliaro, Luci ed ombre nella riforma del processo tributario, cit., p. 2931-2932; M. Conigliaro, Restyling di reclamo-mediazione e conciliazione giudiziale per puntare sugli strumenti deflattivi, cit., p. 3029; P. Sandulli, La Corte costituzionale analizza la mediazione tributaria, in Riv. dir. proc., n. 3/2015, p. 817. Inoltre, cfr. M. Conigliaro, Nuove regole per reclamo e mediazione tributaria, ma la terzietà rimane una chimera, cit., p. 4020, secondo cui «ancora una volta è mancato il coraggio di agire in modo determinato, provando ad introdurre un soggetto che - terzo rispetto alle parti - aiutasse i contendenti a definire bonariamente la controversia ed evitare il contenzioso». 
il reclamo/mediazione dinanzi a funzionari della Pubblica Amministrazione, piuttosto che di fronte a soggetti neutrali ed imparziali.

In tale ottica, è stata ravvisata l'opportunità di affidare l'esame del reclamo ad un ufficio esterno rispetto alla struttura amministrativa che ha redatto l'atto oggetto di contestazione, in modo da assicurarne una "revisione" quanto più oggettiva, idonea a rivedere le determinazioni precedentemente assunte, conformandosi a quanto prospettato dal contribuente ${ }^{26}$.

L'orientamento non può essere condiviso in quanto omette di considerare che l'esigenza di terzietà dell'organo preposto, necessaria nella mediazione civile, non lo è in campo tributario, in cui la procedura di reclamo/mediazione assume natura amministrativa e non giurisdizionale; piuttosto, il legislatore ha considerato sufficiente mantenere l'autonomia, all'interno dell'ente, del soggetto chiamato a decidere sul reclamo, al fine di consentire un corretto esercizio del relativo potere.

Dunque, ogni ente impositore, compatibilmente con la struttura organizzativa di cui è dotato, provvede all'esame del reclamo e della proposta di mediazione mediante apposite articolazioni appartenenti alla propria organizzazione, diverse ed autonome rispetto a quelle che hanno curato l'istruttoria degli atti reclamabili.

Si ritiene che l'istituto non contrasti con il principio d'indisponibilità della pretesa giudiziaria ${ }^{27}$, in quanto, da un lato, rimane nella facoltà dell'ente impositore respingere la proposta di mediazione allorquando possa creare pregiudizio all'erario e, dall'altro, è compito degli uffici fiscali assicurare l'esatta percezione delle entrate tributarie ottemperando al principio di economicità dell'azione amministrativa, suscettibile, però, di creare contrasti con il carattere

${ }^{26}$ Cfr. U. Perrucci, Brevi annotazioni sulla nuova versione del processo tributario, cit., p. 1475.

27 Sulla problematica relativa alla presunta disponibilità/indisponibilità dell'obbligazione tributaria, [quest'ultimo principio desumibile da una serie di norme costituzionali (artt. 23, 53 e 97 Cost.) e volto a limitare i margini di discrezionalità dell'amministrazione finanziaria circa la possibilità di assoggettare a regimi fiscali differenziati contribuenti che esprimono identica capacità contributiva], cfr. G. Tremonti, Imposizione e definitività nel diritto tributario, Milano, 1977, p. 521; M. Redi, Appunti sul principio di indisponibilità del credito tributario, in Dir. e prat. trib., 1995, I, p. 407 ss.; M. Versiglioni, Accordo e disposizione nel diritto tributario. Contributo allo studio dell'accertamento con adesione e della conciliazione giudiziale, Milano, 2001; F. Gallo, La natura giuridica dell'accertamento con adesione, in Riv. dir. trib., 2002, p. 435 ss.; A. Fantozzi, Il diritto tributario, $3^{\text {a }}$ ed., Torino, 2003, p. 274 ss.; A. Cuva, Conciliazione giudiziale ed indisponibilità dell'obbligazione tributaria, Padova, 2007, p. 22 ss.; G. Falsitta, Natura e funzione dell'imposta, con speciale riguardo al fondamento della sua "indisponibilità", in S. La Rosa (a cura di), Profili autoritativi e consensuali del diritto tributario, Milano, 2008, p. 45 ss.; A. Fedele, Autonomia negoziale e regole privatistiche nella disciplina dei rapporti tributari, in S. La Rosa (a cura di), Profili autoritativi e consensuali del diritto tributario, cit., p. 128 ss.; P. Russo, Indisponibilità del tributo e definizioni consensuali delle controversie, in Rass. trib., 2008, p. 595; S. La Rosa, Principi di diritto tributario, Torino 2009, p. 241 ss.; A. Guidara, Indisponibilità del tributo e accordi in fase di riscossione, Milano, 2010, p. 61 ss. 
indisponibile del credito erariale ${ }^{28}$. In realtà, a quest'ultimo dogma non è più possibile attribuire la rigidità di un tempo, essendo stato superato, nei fatti, dalle ipotesi stabilite dalle singole leggi d'imposta, che consentono di rimodulare il tendenziale carattere indisponibile l'obbligazione tributaria ${ }^{29}$.

6. In ordine alle modalità procedurali, la norma prescrive che il ricorso produce anche gli effetti di un reclamo e può contenere una proposta di mediazione con rideterminazione dell'ammontare della pretesa: per ottemperare al rimedio, è necessario redigere un atto processuale, regolato dagli artt. 18 ss., d.lgs. n. 546/1992, i cui requisiti corrispondono a quelli di un ordinario ricorso tributario e la cui peculiarità è quella di realizzare, accanto agli effetti sostanziali e processuali tipici di quest'atto, anche quelli propri della procedura di reclamo/ mediazione $^{30}$. Inoltre, il ricorso produttivo degli effetti tipici del reclamo può contenere anche una proposta di mediazione, volta a definire in maniera stragiudiziale la controversia, attraverso una diversa quantificazione dell'entità del tributo ${ }^{31}$.

Affinché l'Amministrazione Finanziaria possa prendere in seria considerazione l'eventualità di addivenire ad una definizione anticipata della res controversa, evitando alea, durata e costi di un eventuale giudizio, è necessario che la proposta di mediazione, presentata dal contribuente, contenga riferimenti ad elementi di fatto o di diritto sconosciuti alla controparte: tale modus operandi consente l'esperimento, anche in questa fase extragiudiziale, di un effettivo contraddittorio tra i due soggetti del rapporto giuridico d'imposta, volto a favorire una definizione anticipata e condivisa della potenziale controversia ${ }^{32}$.

La mancata osservanza della procedura è sanzionata con la declaratoria di improcedibilità dell'atto introduttivo del giudizio ${ }^{33}$ : il reclamo/ricorso, dopo essere stato ritualmente notificato all'amministrazione finanziaria, dà vita al procedimento di mediazione, che deve necessariamente concludersi nei successivi novanta giorni, a prescindere dalla presenza o meno di una proposta.

${ }^{28}$ Cfr. M. Cicala e A.A. Genise, Osservazioni critiche sulle misure per la revisione della disciplina del contenzioso tributario, in Il fisco, n. 35/2015, p. 3358.

${ }^{29}$ Cfr. M. Conigliaro, Luci ed ombre nella riforma del processo tributario, cit., p. 2932.

${ }^{30}$ Cfr. Circolare Agenzia delle Entrate, 29 dicembre 2015, n. 38/E, cit., p. 33; M. Villani, La parziale riforma del processo tributario, cit., p. 17.

${ }_{31}$ Cfr. S. La Rocca, Il reclamo e la mediazione nel "nuovo" processo tributario, cit., p. 1697; M. Conigliaro, Restyling di reclamo-mediazione e conciliazione giudiziale per puntare sugli strumenti deflattivi, cit., p. 3031.

32 Cfr. S. La Rocca, Il reclamo e la mediazione nel "nuovo" processo tributario, cit., p. 1697. Sul punto, cfr. Circolare Agenzia delle Entrate, 29 dicembre 2015, n. 38/E, cit., p. 34, laddove si puntualizza che «nell'ambito delle controversie relative alle operazioni catastali, tale proposta potrà avere ad oggetto, ad esempio, la modifica del classamento o della rendita catastale determinati dall'Ufficio».

${ }^{33}$ Cfr. V. Uckmar, Interventi sul contenzioso tributario, cit., p. 986; M. VILLANI, La parziale riforma del processo tributario, cit., p. 17. 
Il deposito dell'atto presso la segreteria dell'organo giudicante, prima che sia spirato il termine, ne produce l'improcedibilità, con l'effetto che la Commissione Tributaria adita deve rinviare la trattazione della causa per consentire l'esame del reclamo. Solo successivamente, in caso di mancato accoglimento del reclamo o in assenza di un accordo di mediazione, si producono gli effetti propri del ricorso, aprendo la strada al compimento degli atti processuali tipici del processo tributario, quali il deposito dell'atto introduttivo, di controdeduzioni, di memorie e documenti, e all'adozione di provvedimenti giudiziali ${ }^{34}$.

Pertanto, la proposizione del ricorso, invece di avviare il processo tributario, dà vita ad una fase amministrativa, tesa ad evitare che la controversia sia portata all'attenzione del giudice, mediante l'utile espletamento della procedura di reclamo/mediazione, la cui durata non può comunque eccedere il termine di novanta giorni.

Questa parentesi amministrativa, all'interno del procedimento giurisdizionale, si colloca in un preciso momento, individuato nell'arco temporale compreso tra l'avvio dell'azione giudiziaria, coincidente con la notifica del ricorso, e l'eventuale instaurazione del giudizio tributario ${ }^{35}$. In tal caso, i termini per la costituzione in giudizio del ricorrente restano sospesi per il tempo concesso per addivenire al reclamo/mediazione.

Dal punto di vista procedimentale, i novanta giorni decorrono dalla data di notifica del ricorso all'ente impositore. Decorsi i novanta giorni dalla notifica del ricorso, senza che la procedura di reclamo/mediazione abbia sortito esiti positivi, il ricorrente potrà costituirsi in giudizio, secondo le modalità e procedure di cui all'art. 22, d.lgs. n. 546/1992, ossia mediante il deposito del ricorso presso la Commissione Tributaria Provinciale competente, nei trenta giorni successivi, termine previsto a pena di inammissibilità, rilevabile in ogni stato e grado del giudizio ${ }^{36}$.

Qualora venga accertata la costituzione in giudizio in data anteriore al termine concesso per esperire il reclamo/mediazione (novanta giorni), la Commissione Tributaria adita provvede a rinviare la trattazione della causa al fine di consentire l'esame del reclamo, che, fino al completamento di tale procedura, non potrà assurgere alla dignità di vero e proprio ricorso ${ }^{37}$.

${ }^{34}$ Cfr. S. La Rocca, Il reclamo e la mediazione nel "nuovo" processo tributario, cit., p. 1697; U. PERRUCCI, Brevi annotazioni sulla nuova versione del processo tributario, cit., p. 1475; M. CONIGLIARO, Nuove regole per reclamo e mediazione tributaria, ma la terzietà rimane una chimera, cit., p. 4023.

${ }_{35}$ Cfr. Circolare Agenzia delle Entrate, 29 dicembre 2015, n. 38/E, cit., p. 35.

${ }^{36}$ Cfr. M. Conigliaro, Nuove regole per reclamo e mediazione tributaria, ma la terzietà rimane una chimera, cit., p. 4024; V. Uckmar, Interventi sul contenzioso tributario, cit., p. 986.

${ }^{37}$ Cfr. S. La Rocca, Il reclamo e la mediazione nel "nuovo" processo tributario, cit., p. 1698; V. Uckmar, Interventi sul contenzioso tributario, cit., p. 986. 
7. Il quinto comma dell'art. 17 bis disciplina gli effetti del mancato accoglimento del reclamo o della proposta di mediazione formulata dal contribuente ${ }^{38}$, prevedendo che l'organo destinatario dell'atto, se non intende accogliere le istanze, è obbligato a formulare d'ufficio una propria proposta, tenendo conto dell'eventuale incertezza delle questioni controverse, del grado di sostenibilità della pretesa e del principio di economicità dell'azione amministrativa ${ }^{39}$.

A tal fine, la questione controversa si considera incerta in mancanza di un orientamento consolidato della giurisprudenza di legittimità o di prassi amministrativa in materia. Il grado di sostenibilità della pretesa fa riferimento alle prove e al grado di fondatezza degli elementi addotti dall'istante. Per economicità dell'azione amministrativa s'intende la possibilità di portare a compimento, in maniera rapida ed efficiente, l'attività amministrativa, nel rispetto dei principi di legalità, imparzialità, pubblicità e trasparenza ${ }^{40}$.

La disposizione assume rilievo perché stabilisce un modus procedendi a cui deve attenersi l'organo deputato a pronunciarsi sul reclamo: a differenza del contribuente, al quale è concessa la mera facoltà di presentare una proposta di mediazione, relativa all'ammontare del tributo, delle sanzioni e degli interessi, l'amministrazione finanziaria è invece obbligata a farlo ${ }^{41}$.

Nonostante l'obbligo dell'ufficio fiscale di presentare una proposta di mediazione, il mancato ottemperamento non riverbera alcun effetto negativo sulla pretesa erariale; anzi, consente di consolidare la res controversa dinanzi al giudice tributario, al quale spetta risolverla. Invero, tale momento di dialogo riveste importanza anche per l'amministrazione finanziaria, a cui viene offerta la concreta possibilità di correggere errori, ripensando alle proprie valutazioni, in modo da evitare situazioni che in giudizio potrebbero compromettere l'intera pretesa impositiva ${ }^{42}$.

In ogni caso, l'accoglimento della proposta di mediazione o la rideterminazione della pretesa eviterà il sorgere della controversia: l'accordo di mediazione dovrà contenere l'indicazione specifica degli importi concordati (tributo, interessi

${ }^{38}$ Sui criteri di valutazione del reclamo/mediazione da parte del soggetto preposto alla procedura, cfr. M. D’episcopo, Criteri di valutazione da parte degli Uffici dell'istanza di reclamo-mediazione, in Corr. trib., n. 12/2013, p. 975 ss.

${ }^{39}$ Cfr. M. Conigliaro, Restyling di reclamo-mediazione e conciliazione giudiziale per puntare sugli strumenti deflattivi, cit., p. 3032; U. Perrucci, Brevi annotazioni sulla nuova versione del processo tributario, cit., p. 1475; M. Conigliaro, Nuove regole per reclamo e mediazione tributaria, ma la terzietà rimane una chimera, cit., p. 4023; Circolare Agenzia delle Entrate, 29 dicembre 2015, n. 38/E, cit., p. 37.

${ }^{40}$ Cfr. Circolare Agenzia delle Entrate, 19 marzo 2012, n. 9/E, in www.agenziaentrate.gov.it; F. RASI, Reclamo e mediazione tributaria: tutto risolto dal legislatore e dalla Corte Costituzionale?, in Dir. e prat. trib., n. 3/2014, p. 558, nt. 20, 21 e 22.

${ }^{41}$ Cfr. S. La Rocca, Il reclamo e la mediazione nel "nuovo" processo tributario, cit., p. 1698.

${ }^{42}$ Cfr. S. La Rocca, Il reclamo e la mediazione nel "nuovo" processo tributario, cit., p. 1698, nt. 23. 
e sanzioni), unitamente alle modalità di versamento degli stessi, comprese le eventuali forme di rateizzazione ${ }^{43}$.

Al contrario, qualora non dovesse pervenirsi ad un accordo, «le parti avranno anticipato elementi della difesa tributaria e, formalizzando proposta e controproposta, avranno probabilmente "indebolito" le proprie posizioni processuali» ${ }^{44}$.

Non può, però, disconoscersi che, anche in tali ipotesi, gli elementi forniti nel corso della mediazione possano costituire oggetto di apprezzamento, positivo o negativo, da parte del giudice, influenzando la decisione della controversia: sarà, dunque, quanto mai opportuno formulare proposte di mediazione, il cui contenuto non rechi pregiudizio alla successiva fase contenziosa, anche facendo tesoro dell'esperienza di altri strumenti deflattivi, quali l'accertamento con adesione e la conciliazione tributaria ${ }^{45}$.

8. Laccordo di mediazione, relativo a controversie concernenti un atto impositivo o di riscossione, si perfeziona con il versamento dell'intera somma o della prima rata, da eseguirsi nei venti giorni successivi alla data di sottoscrizione ${ }^{46}$.

Fanno eccezione alla regola generale talune ipotesi in cui la mediazione si perfeziona con la mera sottoscrizione dell'accordo: si pensi, alle controversie relative ai rimborsi d'imposta, il cui accordo di mediazione deve indicare le somme dovute con i termini e le modalità di pagamento ${ }^{47}$.

Un ulteriore esempio è costituito dalle controversie aventi ad oggetto il classamento o la rendita catastale degli immobili, in cui, si procede all'aggiornamento degli atti catastali solo dopo la sottoscrizione dell'accordo di mediazione, nei termini risultanti dal medesimo ${ }^{48}$.

Al pari di altri istituti deflattivi del contenzioso, il perfezionamento dell'accordo consente al contribuente di beneficiare di un trattamento di favor in

${ }_{43}$ Cfr. Circolare Agenzia delle Entrate, 29 dicembre 2015, n. 38/E, cit., p. 38.

${ }_{44} \mathrm{M}$. Conigliaro, Nuove regole per reclamo e mediazione tributaria, ma la terzietà rimane una chimera, cit., p. 4023.

${ }^{45}$ Cfr. M. Conigliaro, Nuove regole per reclamo e mediazione tributaria, ma la terzietà rimane una chimera, cit., p. 4023-4024.

${ }^{46}$ Cfr. M. Conigliaro, Restyling di reclamo-mediazione e conciliazione giudiziale per puntare sugli strumenti deflattivi, cit., p. 3032; U. Perrucci, Brevi annotazioni sulla nuova versione del processo tributario, cit., p. 1475; V. Uckmar, Interventi sul contenzioso tributario, cit., p. 987; M. Conigliaro, Nuove regole per reclamo e mediazione tributaria, ma la terzietà rimane una chimera, cit., p. 4024; M. Villani, La parziale riforma del processo tributario, cit., p. 18; Circolare Agenzia delle Entrate, 29 dicembre 2015, n. 38/E, cit., p. 38.

${ }^{47}$ Cfr. M. Conigliaro, Nuove regole per reclamo e mediazione tributaria, ma la terzietà rimane una chimera, cit., p. 4024; V. Uckmar, Interventi sul contenzioso tributario, cit., p. 987; M. Villani, La parziale riforma del processo tributario, cit., p. 18.

${ }^{48}$ Cfr. Circolare Agenzia delle Entrate, 29 dicembre 2015, n. 38/E, cit., p. 38; M. Conigliaro, Restyling di reclamo-mediazione e conciliazione giudiziale per puntare sugli strumenti deflattivi, cit., p. 3032; U. Perrucci, Brevi annotazioni sulla nuova versione del processo tributario, cit., p. 1475. 
ordine all'entità delle sanzioni amministrative, che si applicano in misura pari al trentacinque per cento del minimo edittale previsto dalla legge. Inoltre, su quanto dovuto a titolo di contributi previdenziali e assistenziali, non sono si fa luogo a sanzioni ed interessi ${ }^{49}$.

\section{Riferimenti Bibliografici}

C. Attardi, Reclamo e mediazione: costituzionalità e ricadute sulla teoria generale del processo tributario, in Corr. trib., n. 18/2013, p. 1446 ss.

B. Bellè, Mediazione e reclamo: due istituti inutili, in Riv. dir. trib., n. 10/2012, pt. I, p. 863 ss.

F. Brighenti, Cartella di pagamento: obbligo di mediazione? (Quando nelle circolari circolano idee confuse), in Boll. trib., n. 9/2014, p. 667.

V. Busa, Le nuove prospettive della mediazione tributaria, in Corr. trib., n. 11/2012, p. 765 ss.

M. Busico, L'ambito di operatività del reclamo e della mediazione: i limiti oggettivi, soggettivi e quantitativi, in Corr. trib., n. 10/2012, p. 704 ss.

S. Capolupo, Mediazione tributaria e accertamento con adesione, in Corr. trib., n. 8/2012, p. 584 ss.

A. CARINCl, Perduranti profili di criticità della presentazione del reclamo, in Corr. trib., n. 37/2012, p. 2877 ss.

M. Chionchio, Z. Gola e R. Lupi, Mediazione tributaria: irragionevolezze vere e presunte sulla via della "deprocessualizzazione", in Lo Stato civile italiano, n. 2/2013, p. 194 ss.

M. Cicala e A.A. genise, Osservazioni critiche sulle misure per la revisione della disciplina del contenzioso tributario, in II fisco, n. 35/2015, p. 3358.

M. Conigliaro, Luci ed ombre nella riforma del processo tributario, in II fisco, n. 30/2015, p. 2931.

M. Conigliaro, Restyling di reclamo-mediazione e conciliazione giudiziale per puntare sugli strumenti deflattivi, in II fisco, n. 31/2015, p. 3030.

M. Conigliaro, Nuove regole per reclamo e mediazione tributaria, ma la terzietà rimane una chimera, in II fisco, n. 42/2015, p. 4021.

G. Corasaniti, Commento all'art. 17-bis, in C. Consolo e C. Glendi, Commentario breve alle leggi del processo tributario, Padova, 2012, p. 236 ss.

G. Corasaniti, Il reclamo e la mediazione nel sistema tributario, Padova, 2013.

A. Cuva, Conciliazione giudiziale ed indisponibilità dell'obbligazione tributaria, Padova, 2007, p. 22 ss.

F. D'ayala Valva, La Corte costituzionale preannuncia le ragioni di illegittimità costituzionale della mediazione tributaria, in Riv. dir. trib., n. 2/2013, pt. II, p. 100.

E. De Mita, I/ reclamo tributario limita i diritti del contribuente, in // Sole 24 Ore, 22 settembre 2013, p. 25.

M. Di Pirro, La mediazione tributaria, Napoli, 2012.

G. Falsitta, Natura e funzione dell'imposta, con speciale riguardo al fondamento della sua "indisponibilità", in S. LA ROSA (a cura di), Profili autoritativi e consensuali del diritto tributario, Milano, 2008 , p. 45 ss.

A. Fantozzi, Il diritto tributario, $3^{a}$ ed., Torino, 2003, p. 274 ss.

A. Fedele, Autonomia negoziale e regole privatistiche nella disciplina dei rapporti tributari, in S. LA ROSA (a cura di), Profili autoritativi e consensuali del diritto tributario, cit., p. 128 ss.

V. Ficari, Brevi note sulla mediazione fiscale, in Boll. trib., n. 19/2012, p. 1364 ss.

V. Ficari, Possibili scenari futuri per la mediazione tributaria, in Corr. trib., n. 40/2013, p. 3187 ss.

E. Fronticelli Baldelli, Effetti del reclamo/mediazione sulla posizione processuale dell'agente della riscossione, in Corr. trib., n. 13/2013, p. 1061 ss.

${ }^{49} \mathrm{Cfr}$. M. Conigliaro, Nuove regole per reclamo e mediazione tributaria, ma la terzietà rimane una chimera, cit., p. 4024; V. Uckmar, Interventi sul contenzioso tributario, cit., p. 987. 
F. Gallo, La natura giuridica dell'accertamento con adesione, in Riv. dir. trib., 2002, p. 435 ss.

C. Gioè, Il reclamo e la mediazione nel giudizio tributario, Torino, 2015.

A. Giovannini, Giurisdizione condizionata e reclamo amministrativo, in Riv. trim. dir. trib., 2012, p. 915 ss.

A. Giovannini, Reclamo e mediazione tributaria: per una riflessione sistematica, in Rass. trib., n. $1 / 2013$, p. 51 ss.

C. Glendi, La riforma del contenzioso tributario, in Dir. e prat. trib., n. 5/2015, pt. I, p. 797.

A. Guidara, Indisponibilità del tributo e accordi in fase di riscossione, Milano, 2010, p. 61 ss.

I. Lucati, II filtro della mediazione obbligatorio anche per le liti tributarie, in La Responsabilità Civile, n. $5 / 2012$, p. 396 ss.

A. Guidara e D. Stevanato, Mediazione fiscale: un provvedimento improvvisato su una strada giusta, in Dial. trib., 2012, 1, p. 92 ss.

S. La rocca, Il reclamo e la mediazione nel "nuovo" processo tributario, in Boll. trib., n. 23/2015, p. 1694.

S. La Rosa, Principi di diritto tributario, Torino 2009, p. 241 ss.

M. Leo, Verso un ordinamento fiscale più moderno, con meno liti e più dialogo, in I/ fisco, n. 29/2015, p. 2809.

M. Leo, La riforma del contenzioso tributario: cose fatte e cose da fare, in II fisco, n. 42/2015, p. 4019.

A. Magliaro, Reclamo e mediazione tributaria: il ruolo del Garante del contribuente, in La Rivista di Finanza, n. 2/2014, p. 39 ss.

C. Maiorano, La mediazione fiscale tra intuizione e realtà, in Boll. trib., 2012, p. 85 ss.

M. Martis, Reclamo e mediazione fiscale. Un nuovo strumento di convergenza tra gli interessi del cittadino e della pubblica amministrazione, in Riv. dir. trib., n. 9/2012, pt. l, p. 809 ss.

S. Mazzocchi, Mediazione tributaria, sospensione cautelare e controversie catastali: un pericoloso "intreccio" di norme, in II fisco, n. 34/2015, p. 3220.

M. Montanari, Il processo tributario nel segno della mediazione, in Dir. e prat. trib., n. 1/2013, pt. I, p. 153 ss.

U. Perrucci, Brevi annotazioni sulla nuova versione del processo tributario, in Boll. trib., n. 21/2013, p. 1475.

F. Pistolesi, Il reclamo e la mediazione nel processo tributario, in Rass. trib., n. 1/2012, p. 65 ss.

F. Pistolesi, Ambito applicativo della mediazione tributaria e sospensione della riscossione, in Corr. trib., n. 19/2012, p. 1429 ss.

G. Pizzonia, Reclamo e mediazione tributaria: deflazione del contenzioso o inflazione di procedimenti?, in Riv. dir. fin., n. 1/2013, p. 71 ss.

F. Rasi, Reclamo e mediazione tributaria: tutto risolto dal legislatore e dalla Corte Costituzionale?, in Dir. e prat. trib., n. 3/2014, p. 558, nt. 20, 21 e 22.

M. Redi, Appunti sul principio di indisponibilità del credito tributario, in Dir. e prat. trib., 1995, I, p. 407 ss.

P. Russo, Indisponibilità del tributo e definizioni consensuali delle controversie, in Rass. trib., 2008, p. 595.

P. Sandulli, La Corte costituzionale analizza la mediazione tributaria, in Riv. dir. proc., n. 3/2015, p. 817.

M. Scuffi, Gli istituti deflattivi del contenzioso tributario secondo la manovra "correttiva" del 2011, in II fisco, n. 47/2011, p. 7641 ss.

G. Sepio, Mediazione tributaria e sanzioni nel coordinamento con gli altri istituti deflativi del contenzioso, in Corr. trib., n. 36/2012, p. 2790 ss.

R. Serrentino, Dubbi di costituzionalità del reclamo e della mediazione tributaria, in Riv. trim. dir. trib., n. 4/2013, p. 929 ss.

R. Serrentino, II "nuovo" istituto della mediazione tributaria: osservazioni critiche, in Dir. e prat. trib., n. 6/2013, pt. I, p. 1347 ss.

G. Tremonti, Imposizione e definitività nel diritto tributario, Milano, 1977, p. 521.

A. Turchi, voce Reclamo e mediazione nel processo tributario, in Dig. disc. priv., sez. comm., Aggiornamento, vol. 6, Torino, 2012, p. 615 ss.

V. Uckmar, Interventi sul contenzioso tributario, in Dir. e prat. trib., n. 6/2015, pt. I, p. 985. 


\section{Versiglioni, Accordo e disposizione nel diritto tributario. Contributo allo studio dell'accertamento con adesione e della conciliazione giudiziale, Milano, 2001. \\ M. Villani, La parziale riforma del processo tributario, in Il tributo, n. 12/2015, p. 17.}

\section{Abstract:}

Il reclamo-mediazione, sintomatico dell'evoluzione del sistema normativo verso una "marginalizzazione" della fase contenziosa, è allocato nella fase che precede l'instaurazione del processo tributario. Per le controversie, ricomprese nella giurisdizione tributaria, il cui valore non ecceda i 20.000,00 euro, è richiesta, ai fini della procedibilità della domanda, la preventiva proposizione di un reclamo, che può anche contenere una proposta di mediazione, volta a dirimere l'insorgenda lite; in caso di mancato accoglimento del reclamo o della proposta di mediazione, si producono gli effetti propri del ricorso tributario. Sotto il profilo oggettivo, l'obbligo del previo esperimento del reclamo ha ad oggetto le sole controversie, rientranti nella giurisdizione tributaria. Dal punto di vista soggettivo, l'ambito di applicazione del reclamo-mediazione concerne tutte le controversie rientranti nella giurisdizione tributaria, aventi ad oggetto l'impugnazione di atti e provvedimenti emessi da qualsiasi tipologia di ente impositore. L'organo competente a conoscere della procedura appartiene alla stessa autorità che ha emanato l'atto impugnato, che vi provvede attraverso strutture diverse ed autonome da quelle che hanno curato l'istruttoria degli atti reclamabili.

Keywords: reclamo; mediazione tributaria; deflazione del contenzioso tributario.

\section{The complatin and mediation between legal proceedings}

\section{Summary}

The complaint/mediation, symptomatic of the evolution of the regulatory system towards a "marginalization" of the litigation phase, is located in the phase preceding the taxation process. For disputes covered by tax jurisdiction whose value does not exceed $€ 20,000.00$, the submission of a claim is required - for the purpose of the applicability of the claim - to contain a proposal of mediation intended to prevent the onset of the legal proceedings; in case of failure to accept the claim or the mediation proposal, the taxation is applied. From an objective point of view, the obligation of the prior exercise of the complaint concerns only the disputes that fall within the tax jurisdiction. From the subjective point of view, the scope of application of the mediation/complaint concerns all disputes within the tax jurisdiction, which concern the appeal of acts and measures provided by any type of taxpayer. The body competent to know the procedure belongs to the same authority that issued the contested measure, thus different and autonomous structures from those who have investigated the acts of reclamation are involved.

Keywords: complaint; tax mediation; reduction of tax disputes. 